EOMmun Communication et organisation

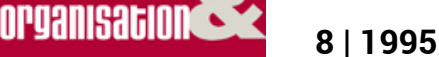

La communication au service de l'information?

\title{
Journalisme et développement
}

Jean-Paul Brunel

\section{OpenEdition}

Journals

Édition électronique

URL : http://journals.openedition.org/communicationorganisation/1809

DOI : 10.4000/communicationorganisation. 1809

ISSN : 1775-3546

\section{Éditeur}

Presses universitaires de Bordeaux

Édition imprimée

Date de publication : 1 novembre 1995

ISSN : 1168-5549

Référence électronique

Jean-Paul Brunel, « Journalisme et développement », Communication et organisation [En ligne], 8| 1995, mis en ligne le 26 mars 2012, consulté le 10 décembre 2020. URL : http://journals.openedition.org/ communicationorganisation/1809; DOI : https://doi.org/10.4000/communicationorganisation.1809

Ce document a été généré automatiquement le 10 décembre 2020.

(C) Presses universitaires de Bordeaux 


\title{
Journalisme et développement
}

\author{
Jean-Paul Brunel
}

1 L'esprit du développement peut-il gagner l'ensemble de la rédaction d'un quotidien régional? Au sein d'une entreprise de presse, les journalistes peuvent-ils, doivent-ils - se considérer - et être considérés - comme des acteurs du développement, voire comme les moteurs de celui-ci.

2 S'interroger ainsi pourrait laisser penser qu'ils sont aujourd'hui indifférents, étrangers, voire tenus éloignés des évolutions éditoriales, commerciales ou industrielles de leur journal. Rien n'est moins exact bien sûr et de Multiples exemples attestent au contraire de leur pleine intégration à la réflexion stratégique au sein des publications. Il reste que les situations sont extrêmement contrastées selon les types de presse, les structures d'entreprise, les modes de management ou enfin, la personnalité ou l'itinéraire de leurs dirigeants.

3 La notion de développement est fort ancienne dans la presse quotidienne régionale, mais elle a longtemps assemblé à la prose de M. Jourdain. Le souci du lecteur et du client, l'adéquation à ses attentes et à ses besoins, les notions de service, de lien social et affectif, ont toujours inspiré sa démarche. Mais son pragmatisme, sa légendaire prudence provinciale sont parfois allés de pair avec une absence de vision du long terme. Cela ne l'a pas empêché de négocier de véritables révolutions technologiques, industrielles et sociales.

4 Mais il est de fait que, longtemps en situation de monopole, bercée par sa prospérité financière et confortée par la fidélité de son lectorat, la PQR s'est souvent consolidée sans esprit de conquête, préférant accroître son taux de pénétration dans ses places fortes plutôt que d'investir dans des politiques audacieuses mais aléatoires d'extension de ses territoires. Les accords de zone limitant les agressions ont permis aux uns et aux autres de construire de véritables citadelles et de favoriser les diversifications. Depuis lors, les années de faible croissance, ponctuées de crises économiques aiguës, l'apparition de concurrences multiples, les évolutions des modes de vie du citoyen-consommateur, l'ont conduit à des révisions déchirantes. Diminution structurelle des recettes publicitaires, érosion de la diffusion dans les centres urbains, vieillissement d'un lectorat qui par 
ailleurs tarde à se renouveler, lui font aujourd'hui obligation non seulement de s'adapter mais surtout d'adopter des stratégies de combat.

Dans cette lutte pour la survie, les journalistes doivent-ils se situer en première ligne? Les éditeurs n'associent encore que trop rarement leur rédaction aux évolutions en cours. Encore qu'il faille se garder de toute généralisation. Au mieux informés, souvent consultés dans des groupes de réflexion alibi, ils assistent en spectateurs frustrés à des changements de formule imposés par des consultants-graphistes sur la base d'études de marketing souvent impulsées par les directions commerciales seules. Ils ont alors beau jeu de prétendre qu'on les tient pour quantité négligeable et de stigmatiser les dérives identitaires d'un journal auquel ils sont souvent par nature viscéralement attachés.

Peut-être devaient-ils s'interroger aussi sur leur état d'esprit passé et se demander notamment s'ils ne sont pas pour une part responsables de cette prétendue marginalisation. Les éditeurs font souvent état du corporatisme étroit de leur rédaction. $\mathrm{Au}$ prétexte du respect de leur indépendance, de leur déontologie

7 Professionnelle ou encore de la défense d'intérêts catégoriels, n'ont-ils pas eux-mêmes contribué à creuser le fossé avec leur hiérarchie confortant celle-ci dans une aimable indifférence, dès lors qu'à l'inverse d'un Syndicat du Livre, ils ne détenaient pas le pouvoir d'empêcher la Parution du journal?

8 On peut comprendre que les journalistes n'aient eu de cesse au fil des années de se protéger de l'influence toujours grandissante des commerciaux, voire de leur intrusion dans des domaines de compétence qui n'étaient Pas les leurs, a vérité est probablement plus complexe.

9 De même que l'on peut reprocher à certains éditeurs d'avoir abandonné depuis longtemps l'identité, les valeurs fondatrices de leur titre et d'avoir renoncé à miser sur la qualité rédactionnelle de leur journal pour asseoir la Progression de sa diffusion; de même on peut faire grief à certains journalistes de ne pas avoir intégré suffisamment tôt «la culture de vente ", d'être restés indifférents à la dimension commerciale de l'entreprise de presse, d'avoir Par suffisance, snobé tout ce qui ne concernait pas l'information.

Les jeunes générations appréhendent fort heureusement les situations autrement. Le monde dans lequel ils ont grandi, leur éducation, leur formation Professionnelle, leur rapport à l'économie et à l'entreprise, ${ }^{\circ}$ t brisé la plupart des tabous.

11 Chez leurs aînés; il se trouve fort heureusement nombreux responsables dans les rédactions et les services commerciaux pour accélérer les décloisonnements, créer les conditions de concertation et de mise en place de synergies. Il existe aussi par bonheur des éditeurs pour additionner les compétences et créer un esprit d'entreprise, au-delà des clivages professionnels et des intérêts corporatistes.

12 Des projets d'entreprises ambitieux dans plusieurs titres aboutissent à une remarquable utilisation des talents, à une mobilisation des énergies autour d'une ambition commune : le développement du journal sur fond d'amélioration de sa qualité.

Cela suppose le respect absolu du préalable suivant : la reconnaissance par l'éditeur du rôle éminent et irremplaçable des journalistes dans la conception et la réalisation du journal ; et la franche délégation octroyée à la rédaction pour mettre en œuvre un projet éditorial sur lequel se fonde toute stratégie de promotion du titre et de commercialisation des espaces publicitaires. Avant d'être un produit, un quotidien régional demeure un journal dont la maîtrise relève sans confusion possible de la compétence des journalistes. 

diffusion et de publicité les plus éclairés. Tel d'entre eux a coutume de dire à ses collègues de la rédaction: «le journal, c'est vous qui le vendez!». Au-delà de la boutade, je ne connais pas meilleur raccourci pour exprimer la primauté de la rédaction dans la mission de développement d'un titre. Mais quelle écrasante responsabilité pour les journalistes et que d'humilité, de renoncement au narcissisme cela suppose. lecteurs en même temps qu'à leurs éditeurs. Que la sanction du lecteur se mesure certes aux chiffres de la diffusion mais relève aussi du «qualitatif » des enquêtes d'audience et des études de marketing. Pour évidente qu'elle soit, cette exigence n'est pas communément admise au sein des rédactions où l'on se protège furieusement des réactions de l'extérieur et, par principe-même, des «vu-lu » et autres entretiens avec les lecteurs-clients. rapidement dans les mentalités sans que personne n'y perde son âme. Et c'est bien évidemment aux chefs d'entreprises de presse d'en garantir les conditions. organisations au travers d'une communication dynamique, forte, sans complaisance et destinée à l'ensemble des collaborateurs de l'entreprise alors même -et c'est un véritable paradoxe dans des entreprises de communication - qu'elle ne transite souvent que par l'encadrement ou des «aiguilleurs » réputés pour leur allégeance ou leur aptitude à la désinformation. la sous-tendent. projet adapté à leurs compétence et à leur fonction. C'est sans doute l'un des Points-clés actuels. Dans les journaux qui n'ont souvent guère modifié leurs organisations depuis leur création, l'organisation obéit à une hiérarchie pyramidale dont les effets n'ont guère été gommés par la décentralisation et l'introduction de l'informatique. De telles structures où les délégations ne sont pas clairement consenties sont à bout de souffle. Elles nuisent à la créativité, restreignent la liberté d'initiative, justifient les cloisonnements et les rivalités stériles. Au même titre que la majorité des entreprises, les journaux se trouvent donc contraints d'inventer des formes de management qui évoluent en même temps que leur stratégie.

20 À Sud Ouest, sous l'impulsion de son président Jean-François Lemoine, la politique de développement s'élabore et s'applique de manière concertée et décentralisée. Dans chaque département, les collaborateurs de l'ensemble des services de l'entreprise sont invités chaque année, à leur niveau, à préparer leur Plan concerté de développement ; on utilise familièrement le sigle « $\mathrm{PCD}$ ».

Il s'agit d'abord de recenser les forces et les faiblesses, de repréciser le positionnement du journal, au crible de multiples critères. Quel journal faisons-nous? Comment est-il perçu? Répondons-nous aux attentes de nos lecteurs et clients? Sommes-nous en phase avec l'identité du département? En quoi constituons-nous un lien entre les habitants de chaque commune du département? Quel est l'état de notre diffusion? Quel est le niveau de nos recettes publicitaires? 

de la diffusion-ventes, sont définies les zones géographiques prioritaires pour le journal. Un examen minutieux du réseau de correspondants est effectué au moyen du logiciel « communes» qui nous permet de mesurer leur activité et le degré de présence de la commune dans l'édition. Des actions concertées sont discutées, planifiées et budgétées à plusieurs niveaux : rédaction, diffusion, publicité. La Direction générale valide ensuite les options retenues par les équipes et l'encadrement. Un suivi du déroulement du plan est effectué par le comité de développement. Un bilan à mi-année a lieu en juin.

De telles méthodes de travail donnent-elles des résultats? Assurément. Elles ont le mérite d'éviter le flou, la dispersion, d'adapter les moyens aux objectifs, de ramener à l'essentiel : la progression de la diffusion. Pour évident que cela paraisse, il n'en fut pas toujours ainsi. Les « PCD » ont d'abord insisté sur l'amélioration de l'image du journal, puis ils ont mis l'accent sur la promotion « globale » du titre sans relier les efforts déployés à la notion de résultat. Aujourd'hui le retour sur investissement s'impose à tous. Finies les opérations de prestige, priorité à la fidélisation du lectorat et à la conquête de nouvelles parts de marché dans des secteurs clairement identifiés comme porteurs. Ainsi la démarche journalistique s'appuie-t-elle sur une volonté ainsi résumée : « un journal au service de ses lecteurs et de ses clients ». Une charte rédactionnelle et graphique, une politique de formation des journalistes, une animation soutenue du réseau des correspondants, un management tout entier centré sur l'optimisation des moyens et la complémentarité des savoir-faire, sont les instruments d'une politique de développement qui, sans effets spectaculaires, permet à Sud Ouest de conforter sa position de deuxième quotidien régional derrière Ouest-France. Chaque membre de l'entreprise connait le prix des efforts à consentir pour atteindre ce résultat. Mais au moins sait-il aujourd'hui inscrire sa propre pratique professionnelle dans une voie bien balisée.

\section{RÉSUMÉS}

Les journalistes prétendent travailler en fonction des attentes supposées de leurs lecteurs ou auditeurs. En ce sens, on pourrait dire qu'ils font naturellement acte de communication afin de mieux faire passer l'information. La crise que traverse la presse, économique mais aussi de confiance, peut faire planer un doute sur l'adéquation entre les propositions des uns et les attentes des autres. N'est-il pas temps pour les journalistes de prendre en considération, tout en l'intégrant dans leur propre logique, les outils de marketing dont disposent les journaux? Le développement de la presse (et d'abord sa survie) n'est-il pas à ce prix ? Exemple du quotidien régional français « Sud Ouest ».

Journalists claim to work according to the supposed expectations of their readers or listeners. Thus it could be said that they are involved naturally in communication in order to get over their information. The crisis that the press is currently undergoing, which is economic but which is also a crisis of confidence, might cause some doubt to be shed on the balance between the propositions made by journalists and the expectations of their readers/listeners-Isn't it time that journalists took into consideration the marketing tools that news papers have at their disposal 
and integrate them into their own logic? Isn't this the price to pay for the development of the press (and first of all, its survival)? The example is the regional daily, « Sud Ouest ».

\section{AUTEUR}

\section{JEAN-PAUL BRUNEL}

Jean-Paul Brunel est rédacteur en chef, chargé du développement du journal « Sud Ouest » de Bordeaux depuis 1993 après en avoir été attaché à la rédaction en chef (1988-89) et rédacteur en chef adjoint (1989-1993). Diplômé de l'IUT de journalisme de Bordeaux.il avait précédemment exercé au Midi-Libre, de 1971 à 1988, dont il a été le responsable de la rédaction parisienne (1983-1988). 